\section{The McCrone Atlas of Microscopic Particles: The Modern Dynamic Particle Reference Resource}

\author{
David A. Wiley \\ McCrone Associates, Westmont, IL \\ dwiley@mccrone.com
}

Microscopists have been identifying particulate matter since the seventeenth century. Reference sets of study slides, identification keys, and even atlases of specific groups of microscopic substances were prepared throughout the eighteenth and nineteenth centuries. During the latter half of the nineteenth century and the early twentieth century, these types of resources grew in volume, but none of them attempted to be a comprehensive source.

In 1967, Dr. Walter C. McCrone and his colleagues changed the practice of microscopy with the publication of The Particle Atlas, Edition I. This single volume first edition, a photomicrographic atlas, illustrated and described 404 substances based on analyses using the polarized light microscope.

The rapid and broad popularity of The Particle Atlas, Edition I paved the way for the expanded six volume second edition of The Particle Atlas completed in 1980. The six volumes included 1022 particle characterizations, technique descriptions, and the inclusion of electron microscopy analyses.

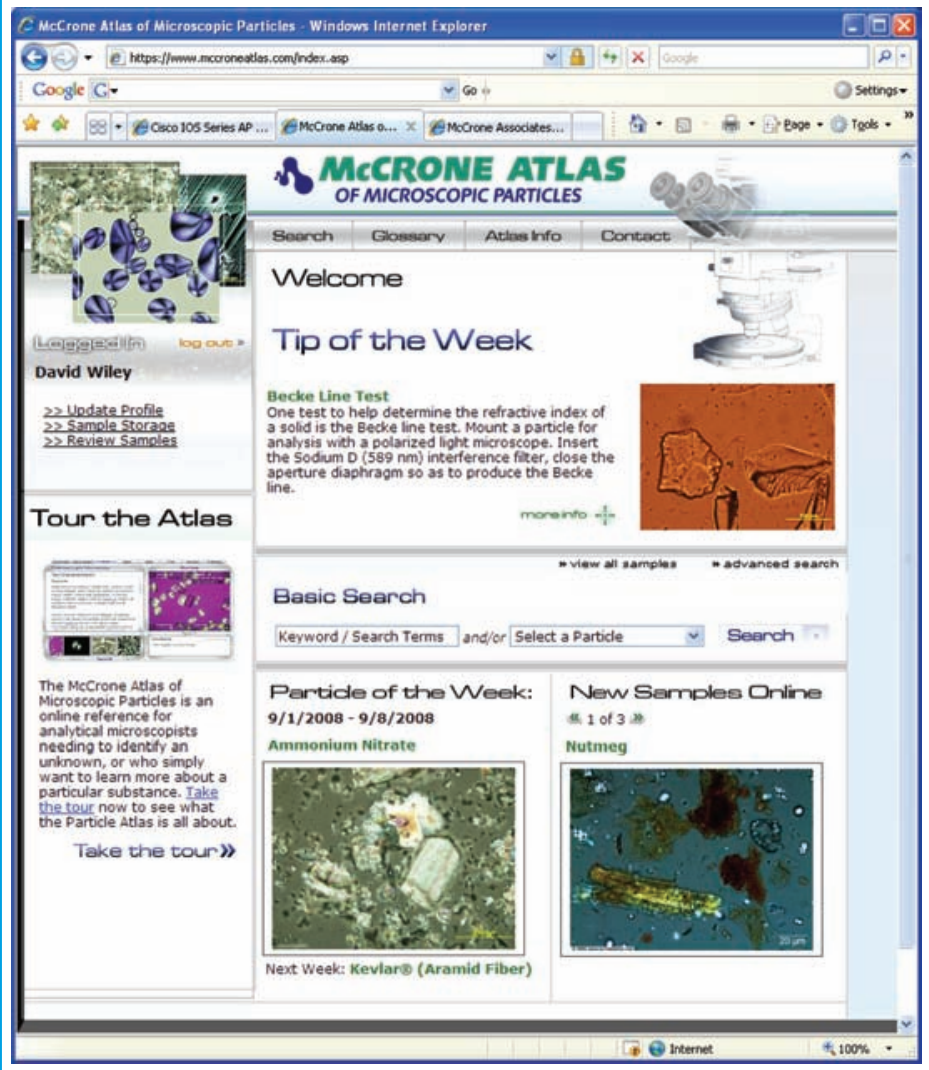

McCrone Atlas of Microscopic Particles home page.

At McCrone Associates, we recognized the critical need for a continuously updated comprehensive reference source for micro-scopists, educators, first responders, forensic scientists, pharmaceutical researchers, and others engaged in materials analysis and identification. We also believed that this new resource should take advantage of the flexibility an of the Internet in order to provide for use in ways never previously available.

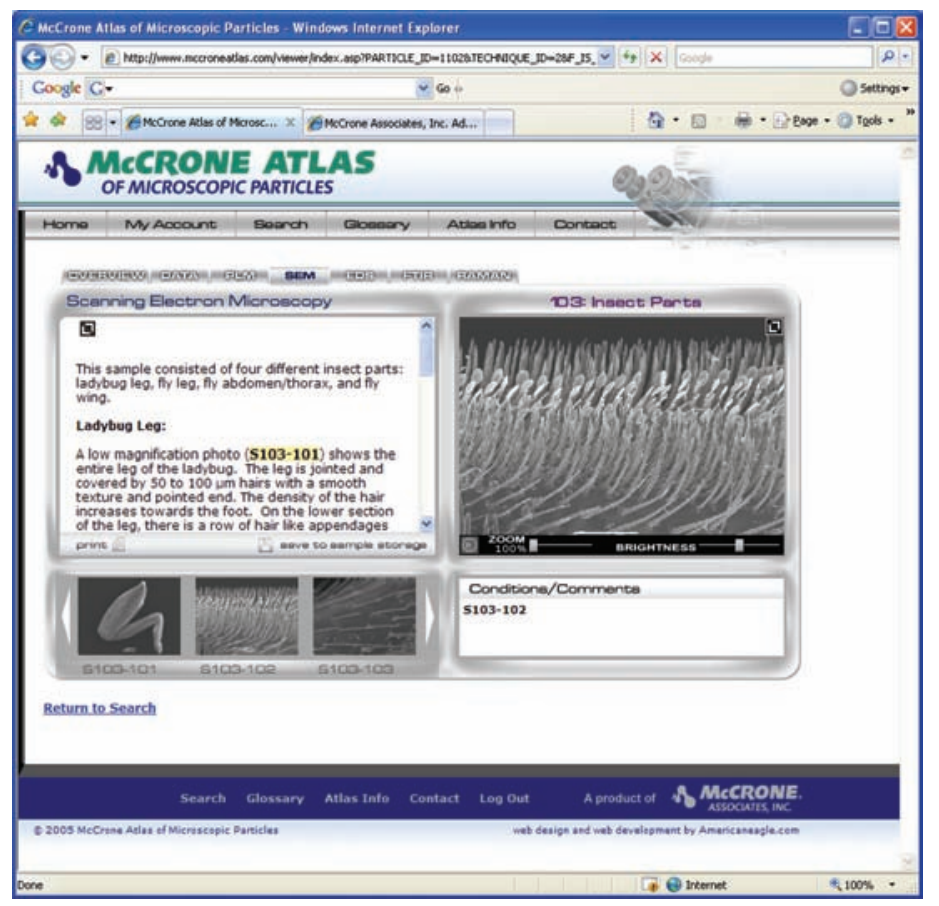

Figure 2. Particle Characterization Viewer: The viewer features analysis tabs located across the top of the viewer, a particle description window on the left with the image thumbnail scroll scrolling viewer below, and the image window on the right with the image detail window below it.

In 2003, development began on a web-based content delivery system that would intuitively display photomicrographs and elec-tron micrographs, spectra, and the characterizations of particulate substances based on modern-day analytical techniques and in-strumentation. Based upon this critical need, John Gustav Delly, one of the original authors and contributors to The Particle Atlas, and a group of microscopists from McCrone Associates began analyzing the first 60 samples for a new web-based interactive atlas. This group of white powders included starches, minerals, metals, and many other materials chosen because of their particular interest to all of our first responder clients. In 2005, McCrone released, for online beta testing, the first batch of samples in a unique, new comprehensive reference resource known as the McCrone Atlas of Microscopic Particles.

The ATLAS combines the knowledge of the world's foremost particle analysts into one of the most comprehensive reference tools ever developed for forensic scientists, first responders, educators, industry and government scientists and researchers. Today, the Atlas includes over 1,000 particle characterizations. During the next several years and beyond, additional characterizations will continue to be posted on a regular basis at www. mccroneatlas.com (Figure 1).

The characterizations include detailed written descriptions along with micrographs and spectra from polarized light microscopy (PLM), scanning electron microscopy (SEM), energy dispersive X-ray spectroscopy (EDS), transmission electron microscopy (TEM), Fourier transform infrared spectroscopy 


\section{Let 4pi take you beyond the capability of any SEM/STEM on the market with}

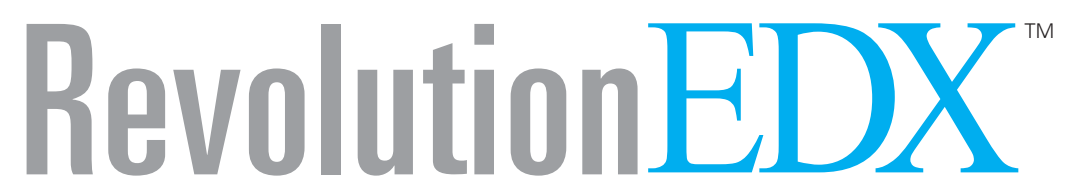

X-ray Microanalysis Systems

Save time and production costs

with 4pi's unique features

Features available exclusively from 4pi:

Event-Streamed Spectrum Imaging ${ }^{\text {Tw }}$

\section{and}

Spatial Frame Lock ${ }^{\text {TM }}$

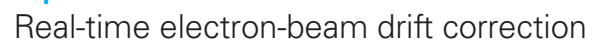
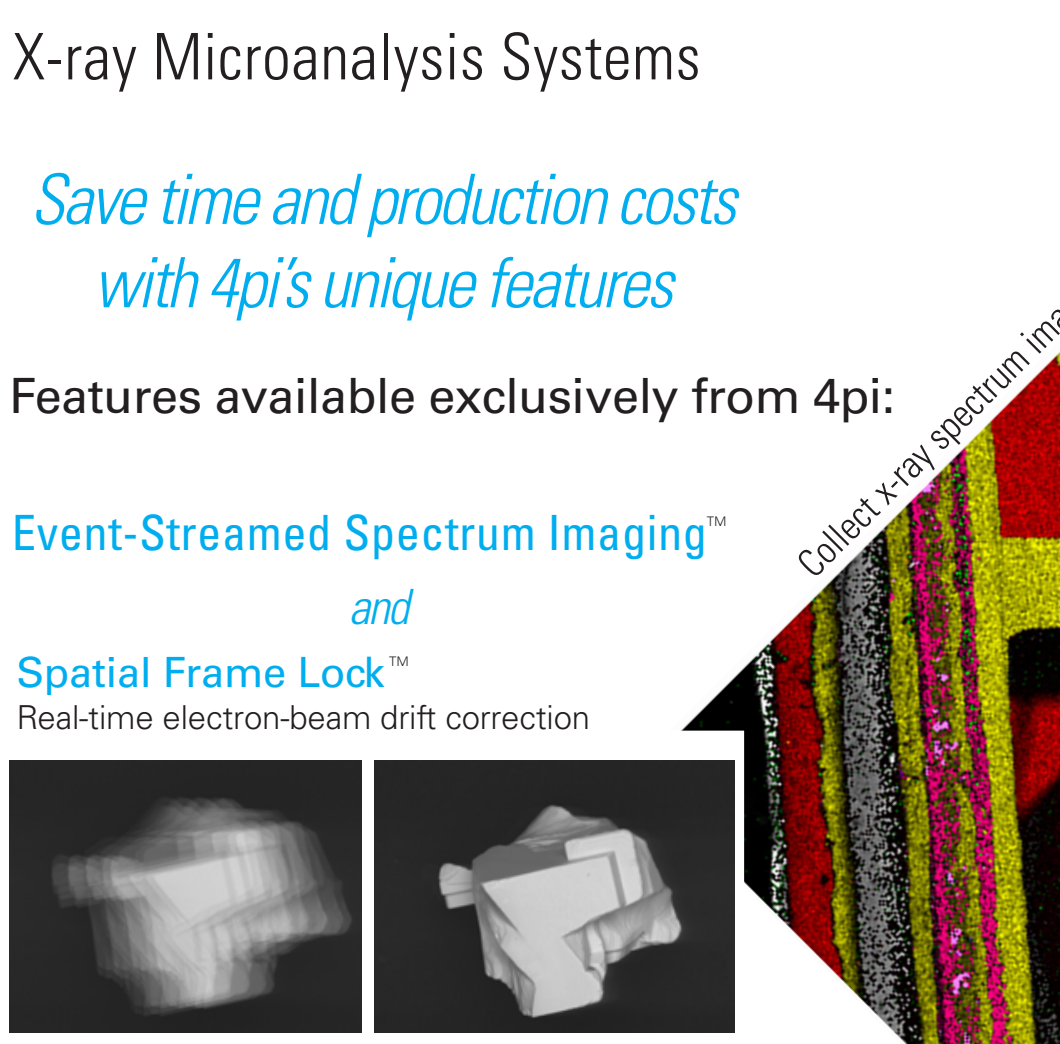


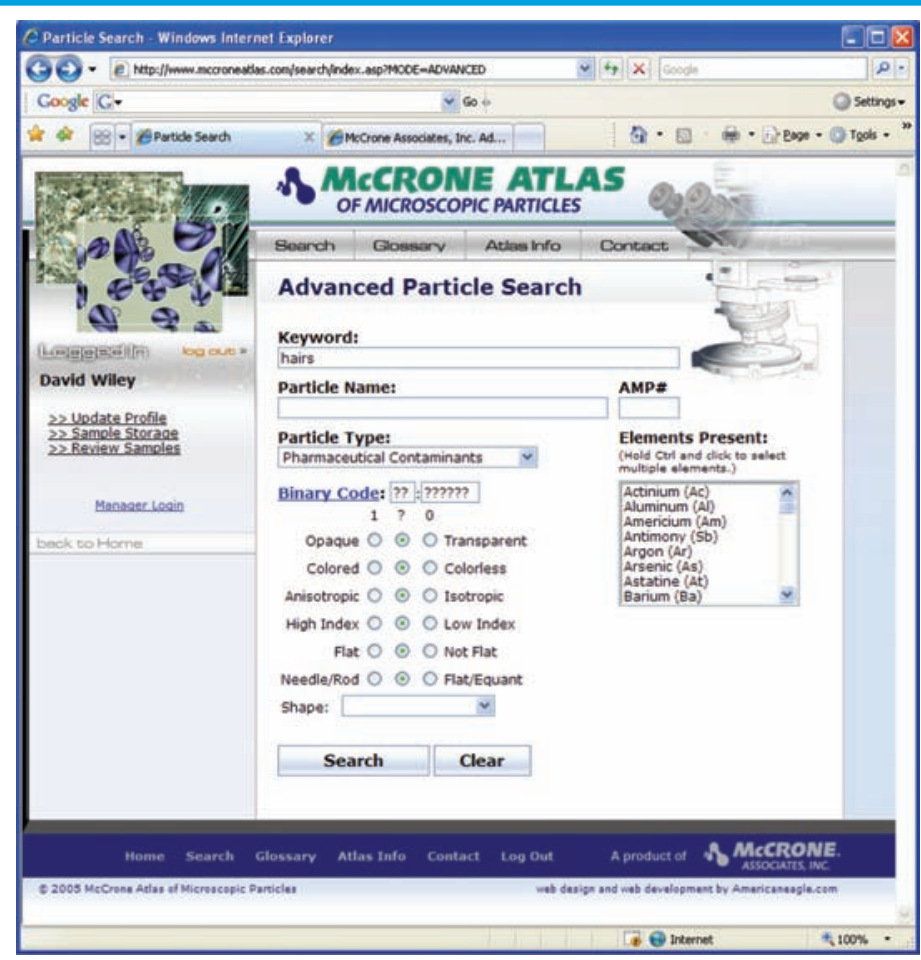

Figure 3. Advanced search page.

(FTIR), Raman spectroscopy, and X-ray diffraction (XRD) analysis. The characterizations are not limited to one or two photomicrographs as they were in the original atlases, but on average each material characterization includes 20 photomicrographs and electron micrographs. Powerful search capabilities allow users to locate the information they need quickly (Figure 2).

The ATLAS home page includes a basic search function allowing users to quickly search and locate particle characterizations of interest. The basic search offers the ability to search by keyword and/or particle type. For more robust searching, we also offer the advanced search page (Figure 3 ) which includes additional search functionality. An advanced search can include any combination of the following search functions-keyword, particle name, particle type, Binary Code, and elements present. The more criteria entered into the search, the more limited the results (Figure 4). There is also a sample index available which lists the characterizations alphabetically by particle name.

Additional features of the online ATLAS include printable particle characterizations for laboratory reporting, full-screen pho-tomicrographs and electron micrographs for comparison to what is being seen through a microscope, interactive spectrum display, particle characterization cross-linking, and personalized sample libraries. Also included among the online ATLAS features is an in-depth glossary of terms, a column highlighting new particles, and microscopy Tips of the Week.

The ATLAS plays a critical role in the first-of-its-kind First Responders Emergency Mobile Response Vehicle designed to help fight the war on terrorism. The vehicle was unveiled in 2006 by the Cleveland Fire Department. The McCrone Group developed new hazardous materials protocols and testing methods for the City of Cleveland Fire Department. This new system of sample collection, transportation, and analyses provides critical timesaving steps for first responders, and permits them to ar- rive at materials identification answers faster than other typical materials-handling protocols.

Additional resources onboard the Mobile Response Vehicle completing the total microscopy solution include fluorescence mi-croscopy for anthrax identification and use of The McCrone Group's online ATLAS. The ATLAS provides first responders with the ability to quickly search and visually compare samples collected at the scene with thousands of stored particle examples within the software/database application-saving minutes and even hours of testing.

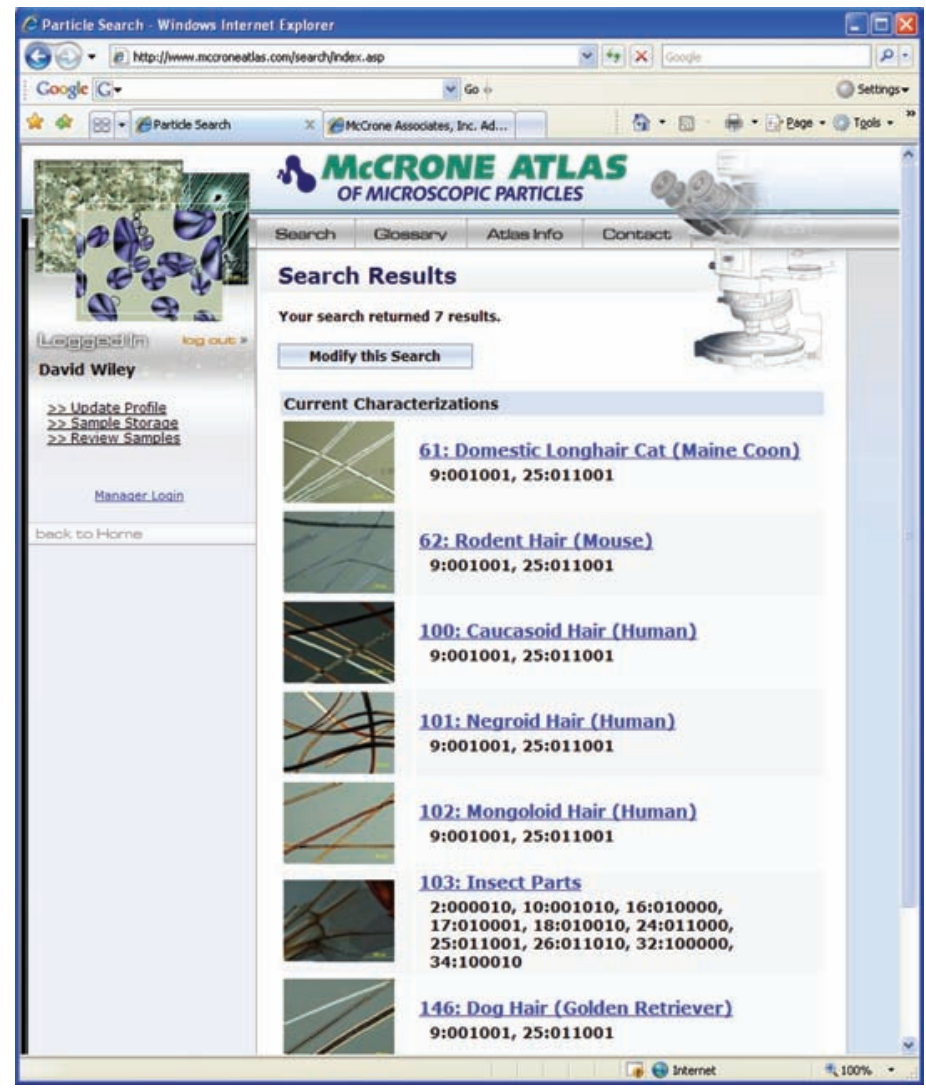

Figure 4. Search Results

While we will continually add new characterizations using all of our analytical capabilities, we thought it would be of great value to subscribers if they had access to the historical characterizations from the original hardcopy version of The Particle Atlas. The historical characterizations will act as place holders in the online database until the original samples are re-characterized using our present-day capabilities. The re-launch of the ATLAS site took place in March 2008 with improved subscription methods, and the addition of 846 historical characterizations from The Particle Atlas volumes two and five.

McCrone Associates is focused on solving the most difficult materials research problems. For over 50 years, we have con-sulted with and solved problems for industrial, academic, and government clients worldwide. Our team of experts uses state-of-the-art instrumentation and techniques to solve particle identification, materials characterization and other analysis problems.

For additional information on the McCrone Atlas of Microscopic Particles, visit www.mccroneatlas.com. 
